\title{
Metallicity diagnostics with infrared fine-structure lines
}

\author{
T. Nagao ${ }^{1,2,3}$, R. Maiolino ${ }^{4}$, A. Marconi ${ }^{5}$, and H. Matsuhara ${ }^{6}$ \\ ${ }^{1}$ Graduate School of Science and Engineering, Ehime University, 2-5 Bunkyo-cho, 790-8577 Matsuyama, Japan \\ e-mail: tohru@cosmos.ehime-u.ac.jp \\ 2 Research Center for Space and Cosmic Evolution, Ehime University, 2-5 Bunkyo-cho, 790-8577 Matsuyama, Japan \\ 3 Optical and Infrared Astronomy Division, National Astronomical Observatory of Japan, 2-21-1 Osawa, 181-8588 Mitaka, Japan \\ 4 INAF - Osservatorio Astrofisico di Roma, via di Frascati 33, 00040 Monte Porzio Catone, Italy \\ 5 Dipartimento di Fisica e Astronomia, Università di Firenze, Largo E. Fermi 2, 50125 Firenze, Italy \\ ${ }^{6}$ Institute of Space and Astronautical Science, Japan Aerospace Exploration Agency, 3-1-1 Yoshinodai, Chuo-ku, \\ 252-5210 Sagamihara, Japan
}

Received 26 July 2010 / Accepted 4 December 2010

\begin{abstract}
Although measuring the gas metallicity in galaxies at various redshifts is crucial to constrain galaxy evolutionary scenarios, only rest-frame optical emission lines have been generally used to measure the metallicity. This has prevented us to accurately measure the metallicity of dust-obscured galaxies, and accordingly to understand the chemical evolution of dusty populations, such as ultraluminous infrared galaxies. Here we propose diagnostics of the gas metallicity based on infrared fine-structure emission lines, which are nearly unaffected by dust extinction even the most obscured systems. Specifically, we focus on fine-structure lines arising mostly from HII regions, not in photo-dissociation regions, to minimize the dependence and uncertainties of the metallicity diagnostics from various physical parameters. Based on photoionization models, we show that the emission-line flux ratio of ([OIII] 51.80+[OIII] 88.33)/[NIII]57.21 is an excellent tracer of the gas metallicity. The individual line ratios [OIII]51.80/[NIII]57.21 or [OIII] $88.33 /[\mathrm{NIII}] 57.21$ can also be used as diagnostics of the metallicity, but they are more strongly dependent on the gas density. The line ratios [OIII] 88.33/[OIII]51.80 and [NII]121.7/[NIII]57.21 can be used to measure and, therefore, account for the dependences on the gas density and ionization parameter, respectively. We show that these diagnostic fine-structure lines are detectable with $\mathrm{Herschel}$ in luminous infrared galaxies out $z \sim 0.4$. Metallicity measurements with these fine-structure lines will be feasible at relatively high redshift $(z \sim 1$ or more) with SPICA, the future infrared space observatory.
\end{abstract}

Key words. galaxies: abundances - galaxies: evolution - galaxies: ISM - HII regions - infrared: galaxies - infrared: ISM

\section{Introduction}

The metallicity of gas and stars in galaxies is one of the most important properties to distinguish various galaxy evolutionary scenarios, because metals are the results of the cumulative starformation activity and gas inflow/outflow history in galaxies. Because the gas metallicity of galaxies is in most cases much easier to measure than the stellar metallicity, past studies on the metallicity of galaxies and its evolution have mostly focused on the metallicity of the gas phase (e.g., McCall et al. 1985; Zaritsky et al. 1994; Pettini et al. 2001). Several extensive and detailed studies have been performed on the gas metallicity in local galaxies, the connection with other galaxy properties and the metallicity evolution through the cosmic epochs (e.g. Tremonti et al. 2004; Savaglio et al. 2005; Erb et al. 2006; Maiolino et al. 2008; Mannucci et al. 2009, 2010). These observational studies have provided strong constraints on evolutionary scenarios (e.g., de Rossi et al. 2007; Kobayashi et al. 2007; Brooks et al. 2007; Finlator \& Davé 2008).

The vast majority of previous studies on the gas metallicity in galaxies used rest-frame optical diagnostics (see, e.g., Nagao et al. 2006a, and references therein). However, these rest-frame optical diagnostics cannot probe the metallicity of the regions affected by significant dust extinction, which is the case for many star-forming galaxies, especially at high redshift. This is because the fraction of dusty galaxies such as ultra-luminous infrared galaxies (ULIRGs) increases as a function of redshift (e.g., Le Floc'h et al. 2005). Hence, optical metallicity studies probably probe only the outer, less extinguished region of star-forming galaxies, which may deviate substantially from the metallicity of inner more active and more obscured regions of star formation. A clear indication of such a mismatch was recently found in high- $z$ submillimeter galaxies by Santini et al. (2010), who used dust-mass measurements to infer a metal content one order of magnitude higher than inferred from optical metallicity diagnostics. If this mismatch applies to a significant fraction of actively star-forming galaxies, this would imply a major revision of the past results on the metallicity evolution in galaxies based on optical diagnostics. In this context we note that even for starbursts that have been well-studied in the optical, a combination of radio and infrared measurements has conclusively demonstrated that some of the most active star-forming sites are optically obscured (e.g., Gorjian et al. 2001; Vacca et al. 2002; Soifer et al. 2008).

The access to far-infrared diagnostics of the gas metallicity would overcome the dust extinction problems that plague optical measurements. Observational studies on gas metallicity exploiting infrared indicators have already been carried out with the Infrared Space Observatory (ISO). Verma et al. (2003) investigated mid-infrared spectra of 12 nearby starburst galaxies and found a strong correlation between gas metallicity and gas excitation, which is also seen in some optical studies (e.g., Nagao et al. 2006a,b; Maier et al. 2006). Garnett et al. (2004) studied 
chemical properties of gas in M 51 based on the $I S O$ data and found that the $\mathrm{C} / \mathrm{O}$ abundance ratio in M51 is consistent with the solar neighborhood value. These infrared-based metallicity studies are expected to progress dramatically by using the ongoing and forthcoming observational facilities such as Herschel, JWST, SPICA, and ALMA. In this context, it is important to investigate model predictions in detail that yield calibrations between the gas metallicity and flux ratios of emission lines at long wavelengths. Motivated by this, we present the results of photoionization model calculations and the theoretical calibrations of gas metallicity diagnostics based on fine-structure emission lines in mid- and far-infrared wavelength ranges.

\section{The method}

In the mid-infrared to sub-millimeter wavelength range, there are many fine-structure emission lines radiated by various ions on various excitation levels. Although some of them arise within HII regions, some others arise in photo-dissociation regions (PDRs) that surround the inner HII regions. For instance the [CII]157.6 emission, which is the strongest emission line in the spectra of most galaxies and therefore detected even in very distant galaxies (e.g. Maiolino et al. 2005; Iono et al. 2006; Maiolino et al. 2009; Hailey-Dunsheath et al. 2010), is mainly from PDRs rather than HII regions (e.g., Crawford et al. 1985; Stacey et al. 1991). Some emission lines arise in both the HII regions and PDRs (e.g., Petuchowski \& Bennett 1993; Heiles 1994; Aannestad \& Emery 2003; Abel 2006). The latter class of lines are the most difficult to model, because they have a more complex dependence on the various physical parameters of the clouds, and therefore are not ideal metallicity diagnostics. Even lines arising mostly from PDR are not optimal to be used as metallicity diagnostics, since modeling PDRs is very challenging, because it involves complex physical mechanisms of gas heating and radiative transport. The modeling of HII regions is much more easy and generally more understood and tested; in these regions the dependence of the line emissivity on the various physical parameters is much simpler than for PDRs. As a consequence, lines arising mostly from HII regions are more suitable candidates to measure the gas metallicity of the interstellar matter. Therefore, it is very important to understand the emissivity distribution of various fine-structure emission lines in gas clouds and, specifically, understand which ones arise mostly from HII regions and which are instead significantly contributed by PDRs. This is possible by using the publicly available photoionization model code Cloudy, as described by Abel et al. (2005, 2006).

We carried out photoionization model calculations with the Cloudy version 08.00 (Ferland et al. 1998, 2008). We assume pressure-equilibrium gas clouds with a plane-parallel geometry. As described below, the results are not significantly different if we assume constant-density clouds instead of pressureequilibrium clouds, as far as only HII regions are considered (see Sect. 3.2). Calculations are stopped at the depth where the fraction of the $\mathrm{H}_{2}$ molecule reaches $50 \%$ of the total hydrogen, when we investigate lines arising both from HII regions and from PDRs. This depth is defined as the outer edge of PDRs, following Abel et al. (2005). On the other hand, calculations are stopped at the depth where the fraction of $\mathrm{H}^{+}$reaches $1 \%$ of the total hydrogen, when the emission lines arising only from HII regions are investigated (Sect. 3.2). We define this depth as the outer edge of HII regions.

Some low-excitation fine-structure lines can arise beyond this outer edge of PDRs (i.e., fully molecular regions surrounding the inner PDRs; see, e.g., Abel et al. 2005). We do not investigate these fully-molecular regions because our main interest in this work is on emission-lines arising within HII regions, as described below.

The free parameters in our calculations are (1) the hydrogen density of a cloud $\left(n_{\mathrm{H}}\right)$ at the irradiated surface of a cloud; (2) the ionization parameter $(U)$, i.e., the ratio of the ionizing photon density to the hydrogen density at the surface of a cloud; (3) the elemental composition of the gas; and (4) the spectral energy distribution (SED) of the photoionizing continuum radiation. We investigate plane-parallel gas clouds with gas densities $n_{\mathrm{H}}=10^{1}-10^{4} \mathrm{~cm}^{-3}$ at the illuminated face (note that the gas density varies as a function of the depth within gas clouds), and ionization parameters $U=10^{-3.5}-10^{-1.5}$. Here the ionization parameter is a dimensionless parameter defined as

$U=\frac{\Phi(\mathrm{H})}{c n_{\mathrm{H}}}=\frac{1}{c n_{\mathrm{H}}} \int_{v_{1}}^{\infty} \frac{F_{v}}{h v} \mathrm{~d} v$

where $c$ is the speed of the light, $\Phi(\mathrm{H})$ is the surface flux of hydrogen-ionizing photons, $F_{v}$ is the surface energy flux of the input radiation, and $v_{1}$ is the frequency of the Lyman edge $\left(h v_{1}=\right.$ $13.6 \mathrm{eV})$.

The adopted solar elemental abundance ratios (in terms of number density, not mass) and gas-phase depletion factors are given for convenience in Table 1. The adopted depletion factors are derived from the literature (e.g., Cowie \& Songaila 1986; Jenkins 1987; Cardelli et al. 1991; see Ferland 2006, for more details). However, there is no broad consensus on those factors and, moreover, they may depend on the gas density (e.g., Spitzer 1985). We do not discuss this problem further in this work, and we simply adopt the depletion factors given in Table 1. For nonsolar metallicities we assume that both the dust model and the depletion factors are unchanged, but the dust abundance is assumed to scale linearly with the gas metallicity. All elements except nitrogen and helium are taken to be primary nucleosynthesis elements. For helium, we assume a primary nucleosynthesis component in addition to the primordial value, that is,

$\frac{n_{\mathrm{He}}}{n_{\mathrm{H}}}=0.0737+0.0293 Z_{\mathrm{gas}} / Z_{\odot}$

(Groves et al. 2004). Nitrogen is assumed to be a secondary nucleosynthesis element above metallicities of 0.23 solar, and a primary nucleosynthesis element at lower metallicities, i.e.,

$\log \frac{n_{\mathrm{N}}}{n_{\mathrm{H}}}=2 \log \frac{Z_{\mathrm{gas}}}{Z_{\odot}}-4.070$ when $Z_{\mathrm{gas}}>0.23 Z_{\odot}$

and

$\log \frac{n_{\mathrm{N}}}{n_{\mathrm{H}}}=\log \frac{Z_{\text {gas }}}{Z_{\odot}}-4.709$ when $Z_{\text {gas }}<0.23 Z_{\odot}$

(Kewley \& Dopita 2002). Note that the behavior of nitrogen as a secondary element (Eq. (3)) is observationally confirmed through the data of HII regions in galaxies (e.g., van Zee et al. 1998; Pilyugin et al. 2003). Chemical evolutionary models of galaxies also predict such a behavior for a wide range of parameters, including variations of the IMF (e.g., Hamann \& Ferland 1993; Chiappini et al. 2003). The validity of Eq. (3) is sometimes questioned at very high metallicities, $Z_{\mathrm{gas}} \geq 5 Z_{\odot}$, which is however beyond the range studied in this work (see, e.g., Nagao et al. 2006b; Jiang et al. 2008). We investigate gas clouds with gas metallicities $Z_{\text {gas }}=0.05,0.2,0.4,1.0,2.0$, and 3.0 times solar metallicity. In the calculations, Orion-type graphite and silicate 
Table 1. Adopted solar elemental abundance ratios and gas-phase depletion factors.

\begin{tabular}{llc}
\hline \hline Element & $n / n_{\mathrm{H}}$ & Depletion factor \\
\hline $\mathrm{H}$ & $1.00 \mathrm{e}+0$ & 1.00 \\
$\mathrm{He}^{a}$ & $1.03 \mathrm{e}-1$ & 1.00 \\
$\mathrm{Li}$ & $2.04 \mathrm{e}-9$ & 0.16 \\
$\mathrm{Be}$ & $2.63 \mathrm{e}-11$ & 0.60 \\
$\mathrm{~B}$ & $6.17 \mathrm{e}-10$ & 0.13 \\
$\mathrm{C}$ & $2.45 \mathrm{e}-4$ & 0.40 \\
$\mathrm{~N}^{a}$ & $8.51 \mathrm{e}-5$ & 1.00 \\
$\mathrm{O}$ & $4.90 \mathrm{e}-4$ & 0.60 \\
$\mathrm{~F}$ & $3.02 \mathrm{e}-8$ & 0.30 \\
$\mathrm{Ne}$ & $1.00 \mathrm{e}-4$ & 1.00 \\
$\mathrm{Na}$ & $2.14 \mathrm{e}-6$ & 0.20 \\
$\mathrm{Mg}$ & $3.47 \mathrm{e}-5$ & 0.20 \\
$\mathrm{Al}$ & $2.95 \mathrm{e}-6$ & 0.01 \\
$\mathrm{Si}$ & $3.47 \mathrm{e}-5$ & 0.03 \\
$\mathrm{P}$ & $3.20 \mathrm{e}-7$ & 0.25 \\
$\mathrm{~S}$ & $1.84 \mathrm{e}-5$ & 1.00 \\
$\mathrm{Cl}$ & $1.91 \mathrm{e}-7$ & 0.40 \\
$\mathrm{Ar}$ & $2.51 \mathrm{e}-6$ & 1.00 \\
$\mathrm{~K}$ & $1.32 \mathrm{e}-7$ & 0.30 \\
$\mathrm{Ca}$ & $2.29 \mathrm{e}-6$ & $1 \mathrm{e}-4$ \\
$\mathrm{Sc}$ & $1.48 \mathrm{e}-9$ & $5 \mathrm{e}-3$ \\
$\mathrm{Ti}$ & $1.05 \mathrm{e}-7$ & $8 \mathrm{e}-3$ \\
$\mathrm{~V}$ & $1.00 \mathrm{e}-8$ & $6 \mathrm{e}-3$ \\
$\mathrm{Cr}$ & $4.68 \mathrm{e}-7$ & $6 \mathrm{e}-3$ \\
$\mathrm{Mn}$ & $2.88 \mathrm{e}-7$ & 0.05 \\
$\mathrm{Fe}$ & $2.82 \mathrm{e}-5$ & 0.01 \\
$\mathrm{Co}$ & $8.32 \mathrm{e}-8$ & 0.01 \\
$\mathrm{Ni}$ & $1.78 \mathrm{e}-6$ & 0.01 \\
$\mathrm{Cu}$ & $1.62 \mathrm{e}-8$ & 0.10 \\
$\mathrm{Zn}$ & $3.98 \mathrm{e}-8$ & 0.25 \\
\hline & &
\end{tabular}

Notes. ${ }^{(a)}$ See the main text for details of the treatments for helium and nitrogen.

grains (Baldwin et al. 1991; Ferland 2006), as well as polycyclic aromatic hydrocarbon (PAH), are included.

The adopted ionizing continua are the starburst 99 constant star-formation model spectra (Leitherer et al. 1999) with an age of $1 \mathrm{Myr}$ and an $\alpha=2.35 \operatorname{IMF}\left(M_{\text {low }}=1 M_{\odot}\right.$ and $\left.M_{\text {up }}=100 M_{\odot}\right)$. We selected these model parameters because we aim to simulate actively star-forming galaxies (such as infrared galaxies and submillimeter galaxies). However, to demonstrate that our results do not depend on the ionizing continuum shape, we will also investigate models adopting SEDs with older ages (Sect. 3.3). The starburst 99 spectra are available for stellar metallicities of $Z_{\text {star }}=0.05,0.2,0.4,1.0$, and 2.0 times solar metallicity, which are shown in Fig. 1. For models with $Z_{\mathrm{gas}}=$ $3.0 Z_{\odot}$, we adopt the starburst99 spectrum with $Z_{\text {star }}=2.0 Z_{\odot}$. The cosmic background emission from radio to $\mathrm{X}$-ray is also included (see Ferland 2006, for details). However, this component does not affect the calculation results significantly. The heating and ionization by cosmic rays are also included, which affects the thermal solutions especially in the PDRs (see, e.g., Ferland \& Mushotzky 1984; Ferland 2006).

\section{Results}

\subsection{Contributions from $\mathrm{H} I \mathrm{I}$ regions and PDRs}

We first investigate from which parts of the clouds the emission lines originate in order to explore optimal emission-line combinations, which can be used as good metallicity diagnostics.

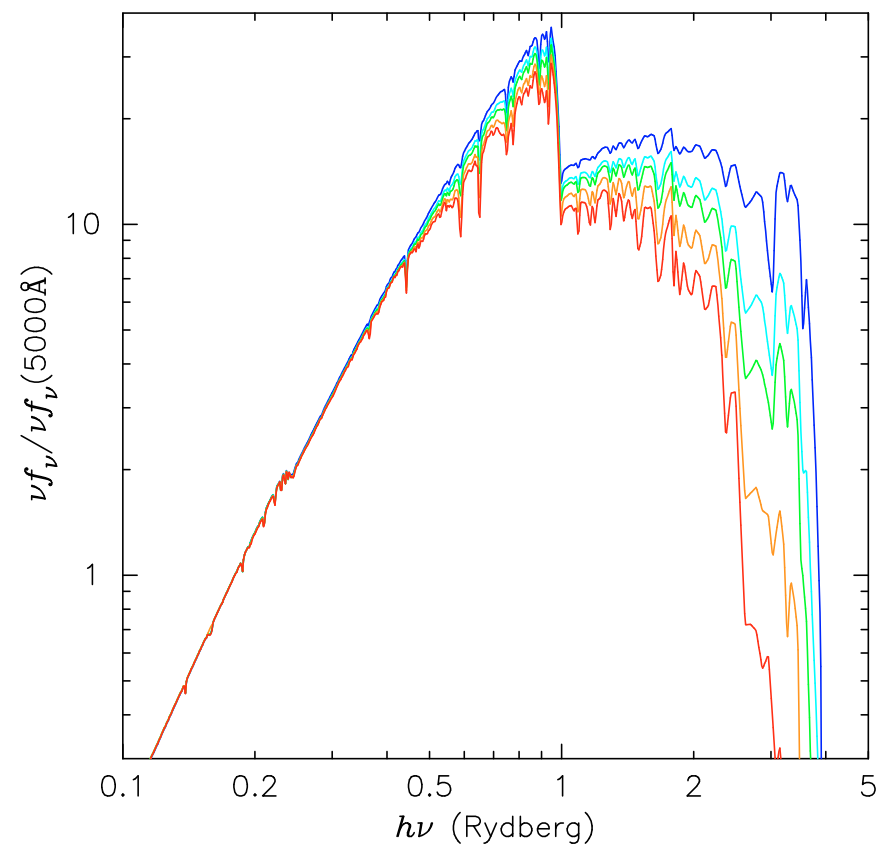

Fig. 1. Spectral energy distributions of the ionizing continuum adopted in our photoionization models, which are starburst99 constant starformation model spectra (Leitherer et al. 1999) with an age of $1 \mathrm{Myr}$ and an $\alpha=2.35 \operatorname{IMF}\left(M_{\text {low }}=1 M_{\odot}\right.$ and $\left.M_{\text {up }}=100 M_{\odot}\right)$. Although most of our analysis adopts these spectra, models with different ages $(3,10$, and $30 \mathrm{Myr})$ are investigated in Sect. 3.3. The blue, cyan, green, orange, and red spectra denote a model with $Z_{\text {star }}=0.05 Z_{\odot}, 0.2 Z_{\odot}$, $0.4 Z_{\odot}, 1.0 Z_{\odot}$, and $2.0 Z_{\odot}$, respectively. The $Z_{\text {star }}=2.0 Z_{\odot}$ spectrum is used also for our $Z_{\text {gas }}=3.0 Z_{\odot}$ calculations (see the main text). All spectra are normalized at $5000 \AA$ ( $\simeq 0.18 \mathrm{Ryd})$.

In Fig. 2 we show hydrogen density structures and cumulative intensities of some fine-structure emission lines of clouds with $Z_{\text {gas }}=0.2 Z_{\odot}$ and $3.0 Z_{\odot}$ as functions of the hydrogen column density. The outer edges of PDRs defined above correspond to the end points of calculations. The outer edges of HII regions are clearly seen as a sharp drop of the density of $\mathrm{H}^{+}$. We show in Fig. 2 that relatively high excitation lines such as [OIII]51.80 and [NIII]57.21 arise within HII regions, while lower excitation lines such as [OI]63.17 and [CII]157.6 arise mostly in PDRs.

To see how HII regions and PDRs contribute to the intensities of various infrared fine-structure emission lines more quantitatively, we give the fraction of the HII region contribution to the total emission-line intensity for the cases of $Z_{\text {gas }}=0.2 Z_{\odot}$ and $3.0 Z_{\odot}, n_{\mathrm{H}}=10^{1.0} \mathrm{~cm}^{-3}$ and $10^{3.0} \mathrm{~cm}^{-3}$, and $U=10^{-2.5}$ and $10^{-1.5}$, in Tables 2 and 3 . The following fine-structure emission lines arise within HII regions without significant contribution from PDRs (at least in the parameter space investigated here): [NeII]12.81, [NeIII]15.55, [SIII]18.67, [SIII]33.47, [NeIII]36.01, [OIII] 51.80, [NIII] 57.21, [OIII]88.33, [NII]121.7, and [NII]205.4 (hereafter "HII region lines"). On the other hand, line intensities of the following low-ionization emission lines are significantly contributed by the PDRs: [SiII]34.81, [OI]63.17, [OI]145.5, and [CII]157.6 (hereafter "PDR lines"). Although the PDR lines arise mostly within PDRs, HII regions also contribute to PDR lines in some cases. Note that the contribution by HII regions given in Tables 2 and 3 for those low-ionization PDR lines are in some cases upper limits. This is because these fine-structure emission lines could also be contributed from molecular cloud regions that extend beyond PDRs (see, e.g., Abel et al. 2005). 


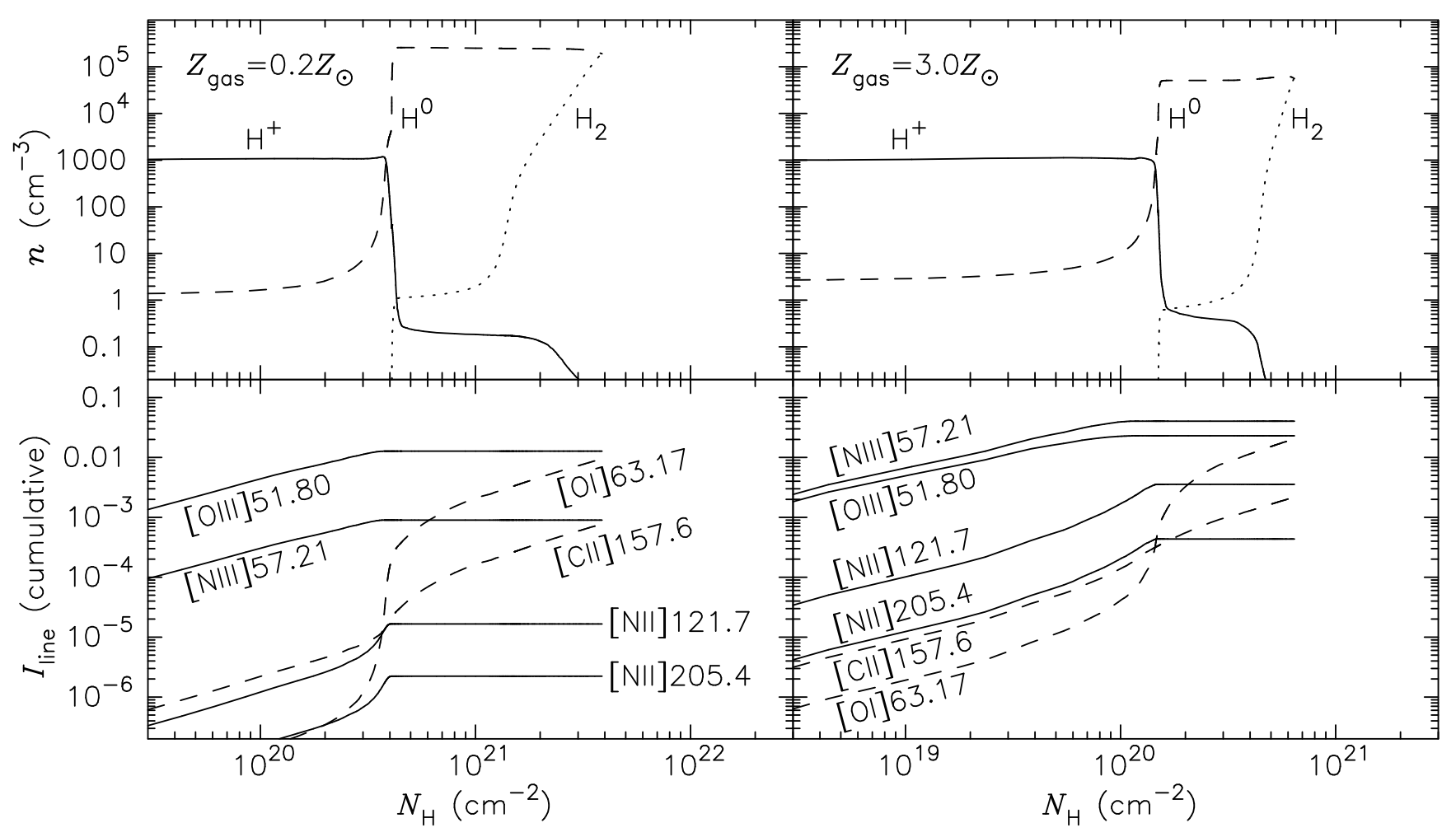

Fig. 2. Hydrogen density structure (upper panels) and cumulative intensity of some fine-structure emission lines (lower panels) of clouds with $Z_{\mathrm{gas}}=0.2 Z_{\odot}$ (left hand panels) and $Z_{\mathrm{gas}}=3.0 Z_{\odot}$ (right hand panels), as a function of the hydrogen column density. For both cases, $n_{\mathrm{H}}=10^{3} \mathrm{~cm}^{-3}$ and $U=10^{-2.5}$ are adopted (where $n_{\mathrm{H}}$ is the hydrogen density at the illuminated face of clouds). Solid, dashed, and dotted lines in the upper panels denote the densities of $\mathrm{H}^{+}, \mathrm{H}^{0}$, and $\mathrm{H}_{2}$, respectively. In the lower panels, solid lines show cumulative intensities for fine-structure emissions mainly from HII regions, while dashed lines are for emissions that are emitted also, or mostly from PDRs.

It is evidently safe to avoid combinations of an HII region line and a PDR line to investigate possible candidate of metallicity-diagnostic flux ratios. We therefore focus only on HII region lines to avoid significant parameter dependences in emission-line flux ratios.

\subsection{Metallicity-sensitive emission-line flux ratios}

Metallicity diagnostics by using infrared fine-structure lines have also been discussed in some previous studies. The main approach consists in calculating abundances of ionic species directly based on their emissivity. The flux $\left(F_{\mathrm{X}^{+i}}\right)$ of a given ionic species $\left(\mathrm{X}^{+i}\right)$ is the product of the density of the ion $\left(n_{\mathrm{X}^{+i}}\right)$, the electron density $\left(n_{\mathrm{e}}\right)$, and the emissivity of the ion $\left(j_{\mathrm{X}^{+}}\right)$. Therefore, in HII regions (where $n_{\mathrm{H}^{+}} \simeq n_{\mathrm{H}}$ by definition), the following relation applies:

$\frac{F_{\mathrm{X}^{+i}}}{F_{\mathrm{H}^{+}}}=\frac{n_{\mathrm{X}^{+i}} n_{\mathrm{e}} j_{\mathrm{X}^{+i}}}{n_{\mathrm{H}^{+}} n_{\mathrm{e}} j_{\mathrm{H}^{+}}}$,

which can be re-written as

$\frac{n_{\mathrm{X}^{+i}}}{n_{\mathrm{H}}}=\left[\frac{F_{\mathrm{X}^{+i}}}{F_{\mathrm{H}^{+}}}\right]\left[\frac{j_{\mathrm{H}^{+}}}{j_{\mathrm{X}^{+i}}}\right]$,

where the emissivities are calculated once the gas temperature is known from the photoionization model. Once ionic abundances are inferred by photoionization models, we can estimate the elemental abundances by applying appropriate ionization correction factors (ICFs). For instance, Verma et al. (2003) derived elemental abundances of Ne from [NeII]12.81 and [NeIII]15.56, Ar from [ArII]6.99 and [ArIII]8.99, and S from both [SIII] 18.71 and [SIV]10.51, by using hydrogen recombination lines such as $\mathrm{Br} \alpha$, for nearby starburst galaxies based on Spitzer spectra (see also Wu et al. 2008). Panuzzo et al. (2003) also investigated the strategy to derive $\mathrm{N}$ abundance by using [NII]121.7, [NIII] 57.21 , and $\operatorname{Br} \alpha$.

However, the requirement of relying hydrogen recombination lines makes this approach highly uncertain. Indeed, the relatively large wavelength separation between hydrogen recombination lines and metallic emission lines (especially for the case of N) is hard to be covered by the same instrument, and the use of different instruments or facilities may introduce significant systematic errors (caused by, e.g., aperture effects and calibration errors) in the estimated metallicities. Moreover, hydrogen recombination lines are sometimes difficult owing to the effect of stellar absorption features. In addition, the wavelength of $\mathrm{Br} \alpha$ is not long enough to avoid significant dust extinction in many cases, since it is known that the amount of extinction toward the most obscured part of star formation in galaxies can reach up to $A_{\mathrm{V}} \sim 100 \mathrm{mag}$ (e.g., Verma et al. 2003). Although Panuzzo et al. (2003) discussed the use of the radio continuum emission instead of hydrogen recombination lines to overcome the above difficulties, the radio continuum generally contributed by synchrotron emission, or even an active galactic nucleus, and therefore the relation between the radio continuum flux and the hydrogen line flux may be complicated. Therefore, "good" metallicity diagnostics should consist of fine-structure emission lines (not of hydrogen recombination lines) at long wavelengths, whose wavelength separation is not exceedingly large.

Liu et al. (2001) investigated N/O elemental abundance ratios of 51 Galactic planetary nebulae (PNs) and proto-planetary nebulae (PPNs) with [OIII] 51.80, [OIII] $] 88.33$, and [NIII] 57.21. 
T. Nagao et al.: Infrared metallicity diagnostics

Table 2. Fractions of the HII region contribution to total emission-line fluxes in low-metallicity cases $\left(Z_{\mathrm{gas}}=0.2 Z_{\odot}\right)$.

\begin{tabular}{|c|c|c|c|c|}
\hline Line & $\begin{array}{c}n_{\mathrm{H}}=10^{1.0} \mathrm{~cm}^{-3} \\
U=10^{-2.5}\end{array}$ & $\begin{array}{c}n_{\mathrm{H}}=10^{1.0} \mathrm{~cm}^{-3} \\
U=10^{-1.5}\end{array}$ & $\begin{array}{c}n_{\mathrm{H}}=10^{3.0} \mathrm{~cm}^{-3} \\
U=10^{-2.5}\end{array}$ & $\begin{array}{c}n_{\mathrm{H}}=10^{3.0} \mathrm{~cm}^{-3} \\
U=10^{-1.5}\end{array}$ \\
\hline [Ne II] 12.81 & 1.00 & 1.00 & 1.00 & 1.00 \\
\hline [Ne III] 15.55 & 1.00 & 1.00 & 1.00 & 1.00 \\
\hline [S III] 18.67 & 1.00 & 1.00 & 1.00 & 1.00 \\
\hline [S III] 33.47 & 1.00 & 1.00 & 1.00 & 1.00 \\
\hline [Si II] 34.81 & 0.99 & 0.80 & 0.23 & 0.05 \\
\hline [Ne III] 36.01 & 1.00 & 1.00 & 1.00 & 1.00 \\
\hline [O III] 51.80 & 1.00 & 1.00 & 1.00 & 1.00 \\
\hline [N III] 57.21 & 1.00 & 1.00 & 1.00 & 1.00 \\
\hline [O I] 63.17 & 0.70 & 0.05 & 0.02 & $<0.01$ \\
\hline [O III] 88.33 & 1.00 & 1.00 & 1.00 & 1.00 \\
\hline [N II] 121.7 & 1.00 & 1.00 & 1.00 & 1.00 \\
\hline [O I] 145.5 & 0.93 & 0.21 & 0.05 & $<0.01$ \\
\hline [C II] 157.6 & 0.33 & 0.03 & 0.03 & $<0.01$ \\
\hline [N II $] 205.4$ & 1.00 & 1.00 & 1.00 & 1.00 \\
\hline
\end{tabular}

Table 3. Fractions of the HII region contribution to total emission-line fluxes in high-metallicity cases $\left(Z_{\text {gas }}=3 Z_{\odot}\right)$.

\begin{tabular}{|c|c|c|c|c|}
\hline Line & $\begin{array}{c}n_{\mathrm{H}}=10^{1.0} \mathrm{~cm}^{-3} \\
U=10^{-2.5}\end{array}$ & $\begin{array}{c}n_{\mathrm{H}}=10^{1.0} \mathrm{~cm}^{-3} \\
U=10^{-1.5}\end{array}$ & $\begin{array}{c}n_{\mathrm{H}}=10^{3.0} \mathrm{~cm}^{-3} \\
U=10^{-2.5}\end{array}$ & $\begin{array}{c}n_{\mathrm{H}}=10^{3.0} \mathrm{~cm}^{-3} \\
U=10^{-1.5}\end{array}$ \\
\hline$[\mathrm{Ne}$ II] 12.81 & 1.00 & 1.00 & 1.00 & 1.00 \\
\hline [Ne III] 15.55 & 1.00 & 1.00 & 1.00 & 1.00 \\
\hline [S III] 18.67 & 1.00 & 1.00 & 1.00 & 1.00 \\
\hline [S III] 33.47 & 1.00 & 1.00 & 1.00 & 1.00 \\
\hline [Si II] 34.81 & 1.00 & 0.96 & 0.61 & 0.37 \\
\hline [Ne III] 36.01 & 1.00 & 1.00 & 1.00 & 1.00 \\
\hline [O III] 51.80 & 1.00 & 1.00 & 1.00 & 1.00 \\
\hline [N III] 57.21 & 1.00 & 1.00 & 1.00 & 1.00 \\
\hline [O I] 63.17 & 0.57 & 0.06 & 0.03 & 0.02 \\
\hline [O III] 88.33 & 1.00 & 1.00 & 1.00 & 1.00 \\
\hline [N II] 121.7 & 1.00 & 1.00 & 1.00 & 1.00 \\
\hline [O I] 145.5 & 0.82 & 0.12 & 0.05 & 0.02 \\
\hline [C II $] 157.6$ & 0.75 & 0.34 & 0.15 & 0.11 \\
\hline [N II] 205.4 & 1.00 & 1.00 & 1.00 & 1.00 \\
\hline
\end{tabular}

They derived the $\mathrm{N}^{2+} / \mathrm{O}^{2+}$ ratio and transformed it into the $\mathrm{N} / \mathrm{O}$ ratio. Here the two [OIII] lines are used to correct for density effects. The combination of these fine-structure emission lines appears to be an optimal set of candidate metallicity diagnostics because all of their wavelength are long enough, and all of them are simultaneously covered by using some observational facilities, such as the Herschel Photodetector Array Camera and Spectrometer (PACS) (although it requires a small redshift of $z \gtrsim 0.08$ to have all lines in band). We therefore focus on this approach and investigate it further below.

In Fig. 3 the predicted [OIII]51.80/[NIII]57.21 flux ratio is shown as a function of the gas metallicity. It is evident that this flux ratio has a strong metallicity dependence, especially in the high-metallicity range $\left(Z_{\mathrm{gas}}>0.2 Z_{\odot}\right)$. However, this flux ratio also displays (smaller) dependences on the ionization parameter and on the gas density, which prevents us to accurately infer the gas metallicity. Figure 4 shows the predicted flux ratios of [OIII] 88.33/[NIII] 57.21 as a function of the gas metallicity. Again we see the density effects on the flux ratio. Note that models with a lower gas density predict larger flux ratios of [OIII] $] 88.33 /[\mathrm{NIII}] 57.21$, which is in an opposite trend from the case of [OIII] 51.80/[NIII]57.21 (Fig. 3).

The diagnostic accuracy significantly improves if the sum of [OIII]51.80 and [OIII] 88.33 is used instead of only [OIII]51.80, as shown in Fig. 5. The density effect on the flux ratio of $([\mathrm{OIII}] 51.80+[\mathrm{OIII}] 88.33) /[\mathrm{NIII}] 57.21$ is small $(\$ 50 \%)$ over the whole range of metallicities. However, there is still a significant dependence on the ionization parameter, especially at high metallicities $\left(Z_{\text {gas }} \gtrsim 2 Z_{\odot}\right.$ ), where it may cause variation of the ratio up to a factor of $\sim 3$. We will compare these model predictions with observational data in Sect. 4.1.

The predicted fluxes of HII region lines are summarized in Table 4, where they are normalized by the [OIII] 51.88 flux. The results do not change significantly when we adopt constant-density models instead of constant-pressure models. More specifically, the differences in the predicted fluxes of the emission lines given in Table 4 between constant-pressure models and constant-density models are less than $20 \%$ in most cases.

Next we focus on how to account for the dependence of the ionization parameter of the flux ratios shown in Figs. 3-5. To estimate the ionization parameter, pairs of ion species of the same element in a different ionization degree have been often used. We thus examine two flux ratios of [NeII]12.81/[NeIII $] 15.55$ and [NII] 121.7/[NIII]57.21 as possible tools to correct for the ionization parameter effect. Note that also [NeII]12.81 and [NII]121.7 are HII region lines (i.e. not from PDRs), as described in Sect. 3.1. In Fig. 6 we show these two emissionline flux ratios as a function of the density-sensitive flux ratio [OIII]51.80/[OIII] 88.33. Clearly, both of the two flux ratios are strongly dependent on the ionization parameter and therefore they can be used to measure $U$ and correct the metallicity diagnostic flux ratio ([OIII]51.80+[OIII] $] 88.33) /[\mathrm{NIII}] 57.21$ 


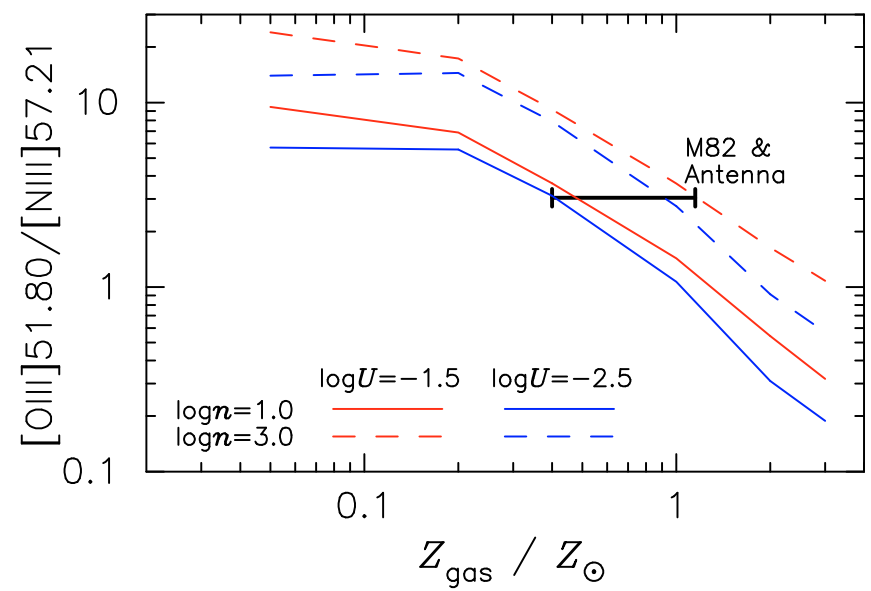

Fig. 3. Predicted emission-line flux ratio of [OIII] $51.80 /[\mathrm{NIII}] 57.21$ as a function of gas metallicity. Blue and red lines denote the models with $U=10^{-2.5}$ and $10^{-1.5}$, and solid and dashed lines denote the models with $n_{\mathrm{H}}=10^{1.0} \mathrm{~cm}^{-3}$ and $10^{3.0} \mathrm{~cm}^{-3}$, respectively. The flux ratio observed in M 82 and in the Antennae galaxy is shown by black horizontal line (these two galaxies show very similar [OIII]51.80/[NIII]57.21 flux ratios; see Table 5). The $x$-range of this horizontal line corresponds to the inferred metallicity range for M 82 and the Antenna galaxy.

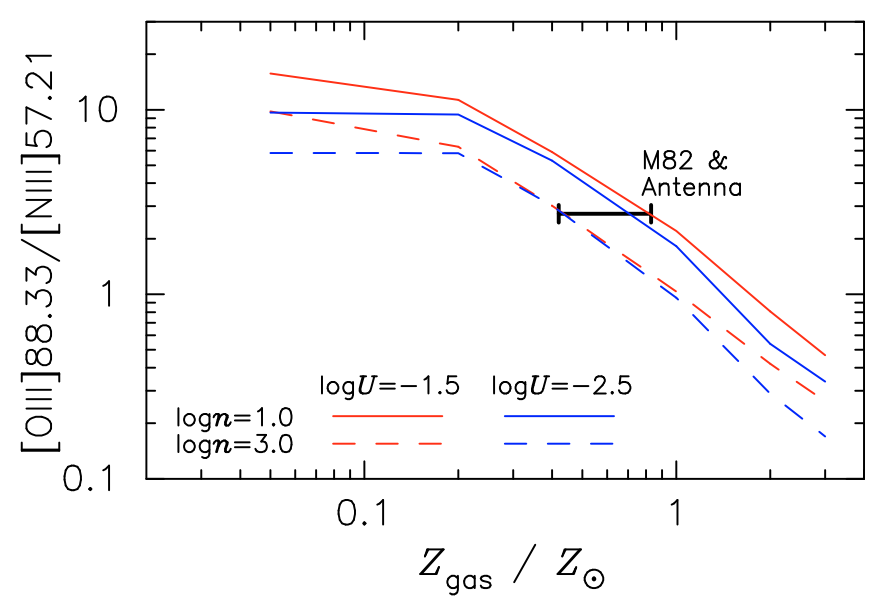

Fig. 4. Same as Fig. 3 but for the flux ratio of [OIII]88.33/[NIII]57.21.

for this dependence. These two flux ratios are also sensitive to metallicity, which is partly due to the metallicity-dependent SED of the ionizing photons (Fig. 1). Therefore an iterative process is required to estimate the metallicity of galaxies by using the diagnostic diagrams given in Figs. 5 and 6. Which is the more appropriate $U$-sensitive flux ratio to correct for the ionization parameter effects on the metallicity determination depends on the specific case. The [NeII]12.81 and [NeIII]15.55 emission lines are moderately strong in star-forming galaxies and have been observed by Spitzer in many galaxies (e.g., Dale et al. 2006), and their flux ratio has only a slight dependence on the gas density, as shown in Fig. 6. However, these lines are more affected by dust extinction effect with respect to the metallicity diagnostic emission lines, [OIII]51.80, [OIII]88.33, and [NIII]57.21. In contrast, [NIII]57.21 and [NII]121.7 do not suffer significant dust extinction. However, the expected emissivity of [NII]121.7 is low, which makes its measurement challenging. In Fig. 7 we also plot the same $U$-sensitive flux ratios, [NeII] 12.81/[NeIII]15.55 and [NII]121.7/[NIII]57.21 as a function of the density-sensitive flux ratio [NII] 121.7/[NII]205.4,

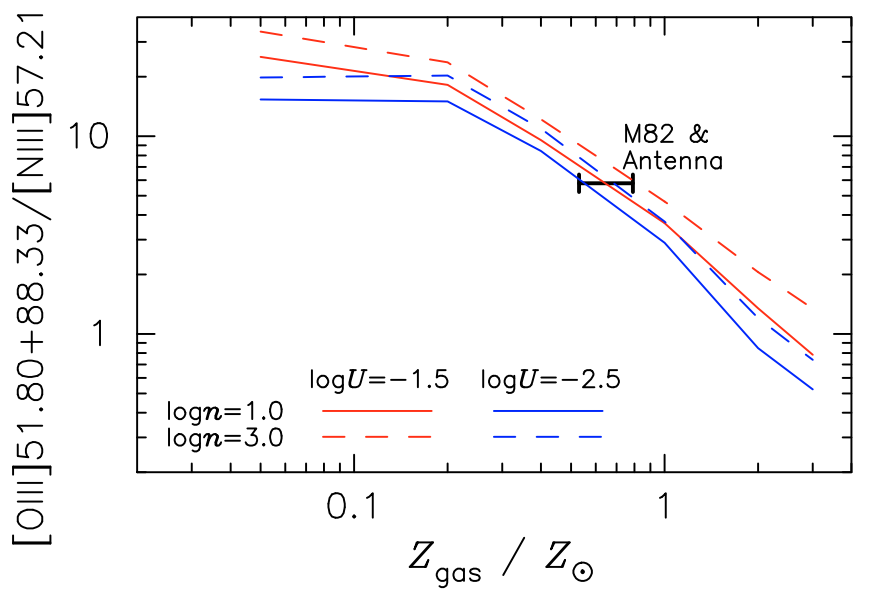

Fig. 5. Same as Fig. 3 but for the flux ratio of ([OIII]51.80+ [OIII] 88.33)/[NIII] 57.21. Note the much lower dependence on the gas density, which makes this ratio particularly suited to measure the gas metallicity.

instead of [OIII] $51.80 /[\mathrm{OIII}] 88.33$. Since the [NII]205.4 emission is very faint and at a very long wavelength, it is very difficult to study with Herschel and SPICA, though its detection should be feasible with ALMA.

\subsection{Dependences on the stellar age}

All emission-line flux ratios shown in Figs. 3-7 are calculated by adopting constant star-formation SEDs with an age of 1 Myr. However, it should be verified whether the predicted flux ratios depend on the age of the stellar population, because the adopted age (1 Myr) seems too young compared to the typical age of star-forming galaxies in general. Cid Fernandes et al. (2003) investigated stellar populations of nearby starburst galaxies and reported that the typical starburst age is $\sim 10^{7.0-7.5}$ yr. More recently, Rodríguez Zaurín et al. (2010) studied stellar populations of low- $z$ ULIRGs, finding similar age ranges. It is therefore important to examine how the emission-line diagnostics studied in this work depend on the age of the stellar populations used for the input SED.

We calculated the emission-line flux ratios of ([OIII]51.80+ [OIII] 88.33$) /[\mathrm{NIII}] 57.21$ and [NII]121.7/[NIII]57.21 by adopting the input SED of stellar populations with ages of 3,10, and $30 \mathrm{Myr}$ in addition to our default value (1 Myr). Figure 8 shows the results of the age dependences of those two diagnostics. The flux ratio of ([OIII]51.80+[OIII]88.33)/[NIII]57.21 changes by less than $30 \%$ in the range $1-30 \mathrm{Myr}$, which is negligible compared with the metallicity dependence of this flux ratio shown in Fig. 5. The age dependence is not significant either for the flux ratio [NII]121.7/[NIII]57.21.

These results are due to the fact that the SED shape significantly changes as a function of the stellar-population age (for the range of $1-30 \mathrm{Myr}$ ) only at $\lambda>3000 \AA$ (see Fig. 8 in Leitherer et al. 1999), when the constant star-formation history is assumed. The SED at shorter wavelengths does not significantly depend on the starburst age, because in that wavelength range it is dominated by emission from massive stars. Since the ionization structure in HII regions is mostly determined by ultraviolet photons (whose SED is not sensitive to the stellar-population age), the flux ratios of HII-region lines are consequently insensitive to the starburst age. These situation may be completely 
T. Nagao et al.: Infrared metallicity diagnostics

Table 4. Predicted emission-line fluxes by photoionization models.

\begin{tabular}{|c|c|c|c|c|c|c|c|c|c|}
\hline \multicolumn{3}{|c|}{ Model Parameter } & \multicolumn{7}{|c|}{ Predicted Fluxes Normalized by $F([\mathrm{OIII}] 51.88)$} \\
\hline $\log n_{\mathrm{H}}{ }^{a}$ & $\log U$ & $Z_{\mathrm{gas}} / Z_{\odot}$ & {$[\mathrm{NeII}] 12.81$} & {$[\mathrm{NeIII}] 15.55$} & {$[\mathrm{OIII}] 51.88$} & {$[\mathrm{NIII}] 57.21$} & [OIII] 88.33 & {$[\mathrm{NII}] 121.7$} & {$[\mathrm{NII}] 205.4$} \\
\hline 1.0 & -1.5 & 0.05 & 0.004 & 0.809 & 1.000 & 0.106 & 1.664 & $\leq 0.001$ & $\leq 0.001$ \\
\hline 1.0 & -1.5 & 0.20 & 0.006 & 0.789 & 1.000 & 0.145 & 1.646 & $\leq 0.001$ & $\leq 0.001$ \\
\hline 1.0 & -1.5 & 0.40 & 0.011 & 0.769 & 1.000 & 0.274 & 1.617 & 0.002 & 0.002 \\
\hline 1.0 & -1.5 & 1.00 & 0.054 & 0.699 & 1.000 & 0.698 & 1.536 & 0.009 & 0.008 \\
\hline 1.0 & -1.5 & 2.00 & 0.394 & 0.396 & 1.000 & 1.845 & 1.488 & 0.078 & 0.055 \\
\hline 1.0 & -1.5 & 3.00 & 0.618 & 0.287 & 1.000 & 3.147 & 1.471 & 0.314 & 0.181 \\
\hline 1.0 & -2.5 & 0.05 & 0.031 & 0.928 & 1.000 & 0.171 & 1.691 & 0.006 & 0.006 \\
\hline 1.0 & -2.5 & 0.20 & 0.053 & 0.916 & 1.000 & 0.179 & 1.693 & 0.007 & 0.007 \\
\hline 1.0 & -2.5 & 0.40 & 0.089 & 0.899 & 1.000 & 0.320 & 1.698 & 0.014 & 0.015 \\
\hline 1.0 & -2.5 & 1.00 & 0.322 & 0.778 & 1.000 & 0.937 & 1.708 & 0.073 & 0.074 \\
\hline 1.0 & -2.5 & 2.00 & 1.382 & 0.409 & 1.000 & 3.229 & 1.737 & 0.598 & 0.543 \\
\hline 1.0 & -2.5 & 3.00 & 1.916 & 0.328 & 1.000 & 5.308 & 1.783 & 1.714 & 1.423 \\
\hline 3.0 & -1.5 & 0.05 & 0.004 & 0.942 & 1.000 & 0.042 & 0.408 & $\leq 0.001$ & $\leq 0.001$ \\
\hline 3.0 & -1.5 & 0.20 & 0.008 & 0.984 & 1.000 & 0.058 & 0.362 & $\leq 0.001$ & $\leq 0.001$ \\
\hline 3.0 & -1.5 & 0.40 & 0.015 & 1.039 & 1.000 & 0.109 & 0.329 & $\leq 0.001$ & $\leq 0.001$ \\
\hline 3.0 & -1.5 & 1.00 & 0.080 & 1.132 & 1.000 & 0.275 & 0.284 & $\leq 0.001$ & $\leq 0.001$ \\
\hline 3.0 & -1.5 & 2.00 & 0.529 & 0.825 & 1.000 & 0.612 & 0.257 & 0.006 & $\leq 0.001$ \\
\hline 3.0 & -1.5 & 3.00 & 0.767 & 0.787 & 1.000 & 0.924 & 0.243 & 0.015 & 0.002 \\
\hline 3.0 & -2.5 & 0.05 & 0.036 & 1.057 & 1.000 & 0.068 & 0.417 & $\leq 0.001$ & $\leq 0.001$ \\
\hline 3.0 & -2.5 & 0.20 & 0.062 & 1.061 & 1.000 & 0.071 & 0.401 & $\leq 0.001$ & $\leq 0.001$ \\
\hline 3.0 & -2.5 & 0.40 & 0.106 & 1.067 & 1.000 & 0.127 & 0.383 & 0.003 & $\leq 0.001$ \\
\hline 3.0 & -2.5 & 1.00 & 0.395 & 0.986 & 1.000 & 0.364 & 0.348 & 0.013 & 0.002 \\
\hline 3.0 & -2.5 & 2.00 & 1.607 & 0.553 & 1.000 & 1.094 & 0.316 & 0.069 & 0.009 \\
\hline 3.0 & -2.5 & 3.00 & 2.245 & 0.534 & 1.000 & 1.757 & 0.298 & 0.155 & 0.019 \\
\hline
\end{tabular}

Notes. ${ }^{(a)}$ Total hydrogen density at the illuminated surface, in units of $\mathrm{cm}^{-3}$.

Table 5. Measured fluxes of infrared fine-structure lines in M 82 and the Antennae galaxy ${ }^{a}$.

\begin{tabular}{lcc}
\hline \hline line & \multicolumn{1}{c}{${\mathrm{M} 82^{b}}^{b}$} & Antenna $^{c}$ \\
\hline$[\mathrm{OIII}] 51.80$ & $10.3 \pm 0.5 \times 10^{-18}$ & $4.9 \pm 0.29 \times 10^{-19}$ \\
{$[\mathrm{NIII}] 57.21$} & $3.4 \pm 0.5 \times 10^{-18}$ & $1.6 \pm 0.058 \times 10^{-19}$ \\
{$[\mathrm{OIII}] 88.33$} & $8.6 \pm 0.4 \times 10^{-18}$ & $4.7 \pm 0.13 \times 10^{-19}$ \\
{$[\mathrm{NII}] 121.7$} & $1.7 \pm 0.3 \times 10^{-18}$ & $<0.44 \times 10^{-19}(3 \sigma)$ \\
\hline
\end{tabular}

Notes. ${ }^{(a)}$ Line fluxes are given in units of $\mathrm{W} \mathrm{cm}^{-2} \cdot{ }^{(b)}$ Data taken from Colbert et al. (1999). ${ }^{\left({ }^{c}\right)}$ Data taken from Fischer et al. (1996).

different for PDR lines such as [CII]157.6, but the age dependence of these PDR lines is beyond the scope of this work. Note that the constant star-formation history is a better assumption than the single-burst model for star-forming galaxies when relatively old ages ( $\sim 30$ Mye) are assumed, because stellar populations with an age of $\sim 30 \mathrm{Myr}$ in the single-burst model do not produce ionizing photons and thus we do not expect detectable emission-line fluxes from these systems. It can be therefore concluded that our analysis on metallicity (and other) diagnostics does not significantly depend on the age of stellar populations.

\section{Discussion}

\subsection{Comparison with observational data}

Here we compare our calculation results on long-wavelength $(\lambda>50 \mu \mathrm{m})$ diagnostic fine structure lines with existing observational data. The only previous instrument that measured the diagnostic fine-structure lines in star-forming galaxies is LWS onboard IS O. Specifically we focus on two galaxies, M 82 and the Antennae galaxy, since many infrared fine-structure lines are measured for them (Fischer et al. 1996; Colbert et al. 1999).
The measured fluxes are summarized in Table 5. Interestingly, the measured flux ratio of [OIII] $51.80 /[\mathrm{OIII}] 88.33$ is quite similar between those two galaxies; 1.2 and 1.0 for M 82 and the Antennae. These flux ratios suggest $n_{\mathrm{H}} \sim 10^{2} \mathrm{~cm}^{-3}$ for $U \lesssim 10^{-2}$ (Fig. 5). The metallicity diagnostic flux ratio of $([\mathrm{OIII}] 51.80+[\mathrm{OIII}] 88.33) /[\mathrm{NIII}] 57.21$ is also similar between M 82 and the Antennae, 5.6 and 6.0 respectively. Taking the inferred gas density into account as well, these flux ratios correspond to $Z \sim 0.5-0.7 Z_{\odot}$, i.e., slightly sub-solar metallicities. The observed flux ratio of [NII]121.7/[NIII]57.21 in M 82 is measured to be 0.5 , which, combined with the our initial metallicity estimation, gives $\log U=-3.5$. Only an upper limit of $[\mathrm{NII}] 121.7 /[\mathrm{NIII}] 57.21<0.28(3 \sigma)$ is reported for the Antennae galaxy, providing an upper limit on $\log U$ of about -3 . Although the constraint on the ionization parameter is not strong, the uncertainty on the ionization parameter does not significantly affect the metallicity estimation.

Although both M82 and the Antennae are well-studied nearby galaxies, it is not so straightforward to compare the inferred gas metallicity based on the fine-structure emission-line flux ratios with that inferred from other metallicity diagnostics, because the aperture size of LWS (the beam-size is $\sim 80$ arcsec in $F W H M$ ) is very large and difficult to compare with other measurements. Origlia et al. (2004) measured the nuclear gaseous and stellar metallicities by using X-ray and near-infrared spectroscopic observations, and they found that both metallicities are close to or slightly less than the solar metallicity in M 82 . Taking the metallicity gradient into account, these metallicities (based also on diagnostics little affected by dust extinction) are consistent with the metallicity inferred through the far-IR fine-structure diagnostics. The situations is similar also for the Antennae galaxy, because its gaseous and stellar metallicities are close to or slightly less than the solar metallicity (Bastian et al. 2006, 2009). Although the comparisons discussed above do not 


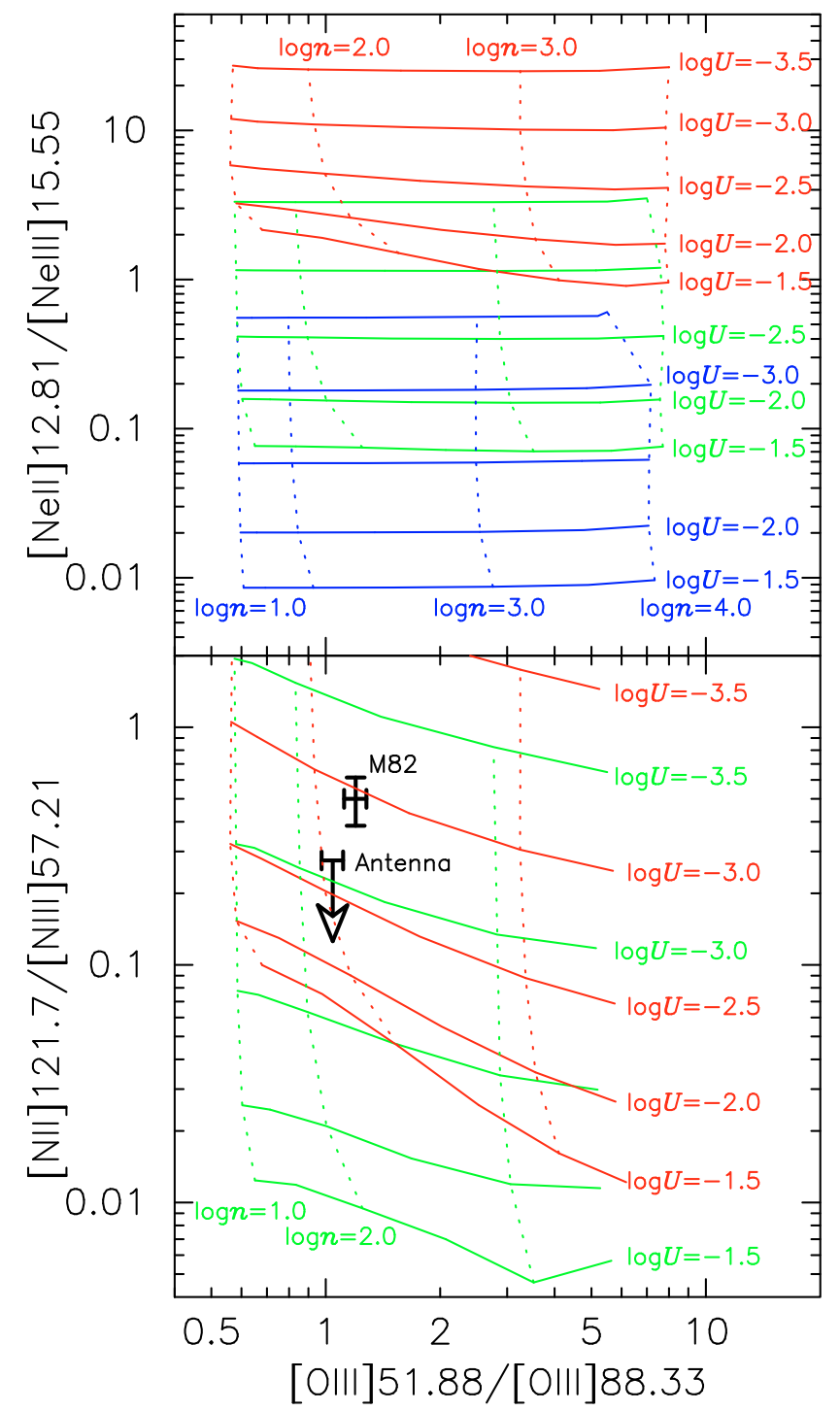

Fig. 6. Predicted emission-line flux ratio of [NeII]12.81/[NeIII]15.55 (upper) and [NII]121.7/[NIII]57.21 (lower) as a function of the densitysensitive flux ratio [OIII]51.80/[OIII] 88.33. Solid and dotted lines denote $U$-constant and $n$-constant model sequences, respectively. Models with $Z_{\mathrm{gas}}=0.2,1.0$, and $3.0 Z_{\odot}$ are denoted by blue, green, and red lines, respectively. The models with $Z_{\text {gas }}=0.2 Z_{\odot}$ or with $n_{\mathrm{H}}=10^{4.0} \mathrm{~cm}^{-3}$ are not shown in the lower panel because [NII]121.7 is extremely faint in those case. Observational data for M 82 and for the Antennae galaxy are shown in the lower panel.

provide a tight test for our new metallicity diagnostics based on far-IR fine structure lines, they do at least suggest a broad consistency with other metallicity diagnostics.

\subsection{Observational feasibility with next-generation instruments}

We finally examine the detectability of the diagnostic finestructure emission lines discussed here with the new-generation instruments, PACS on Herschel (Poglitsch et al. 2010) and SAFARI (Swinyard 2008; Swinyard et al. 2009), the spectrometer planned for SPICA (a 3 m-class cooled telescope in space, Nakagawa 2009). Figure 9 shows the detectability of [NII]121.7, [NIII]57.21, [OIII]51.80, and [OIII]88.33 with PACS. The figure specifically shows the minimum far-infrared luminosity of

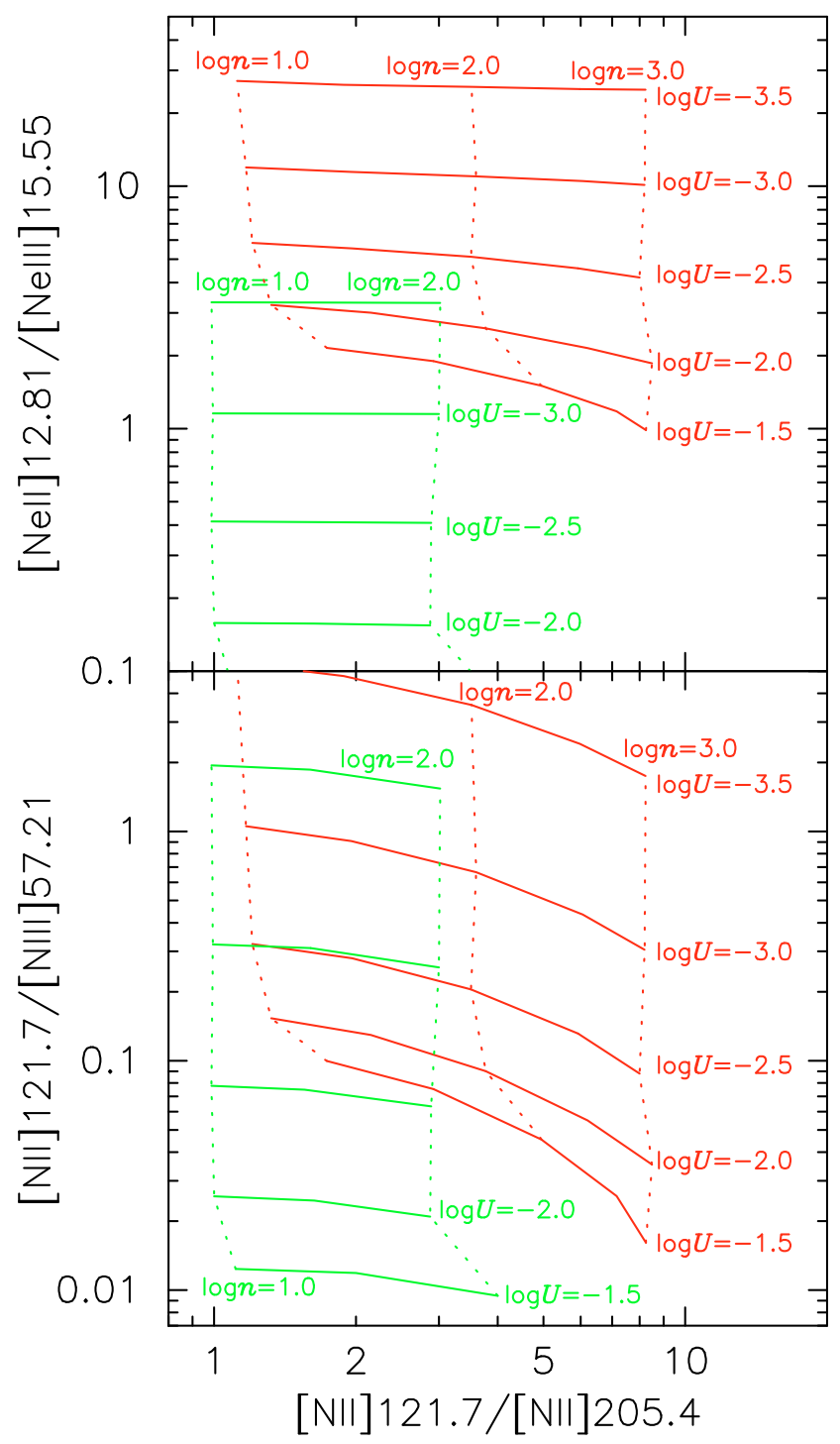

Fig. 7. Predicted emission-line flux ratio of [NeII]12.81/[NeIII $] 15.55$ (upper) and [NII]121.7/[NIII]57.21 (lower) as a function of the densitysensitive flux ratio [NII]121.7/[NII]205.4. Solid and dotted lines denote $U$-constant and $n_{\mathrm{H}}$-constant model sequences, respectively. Models with $Z_{\text {gas }}=1.0 Z_{\odot}$ and $3.0 Z_{\odot}$ are denoted by green and red lines, respectively. Only the models with $n_{\mathrm{H}} \leq 10^{2} \mathrm{~cm}^{-3}$ are plotted for $Z_{\mathrm{gas}}=$ $1.0 Z_{\odot}$, and models with $n_{\mathrm{H}} \leq 10^{3} \mathrm{~cm}^{-3}$ are plotted for $Z_{\mathrm{gas}}=3.0 Z_{\odot}$. This is because the [NII] lines are faint in clouds with a high density owing to the collisional de-excitation effects.

galaxies to have each far-IR line detected $(S / N=5)$ with a $3 \mathrm{~h}$ exposure as a function of redshift. Here we make the simplifying assumption that the luminosity of these emission lines scales roughly with far-infrared luminosity and keep a ratio similar, on average, to what is observed in some nearby galaxies where all of these transitions were observed with past ISO

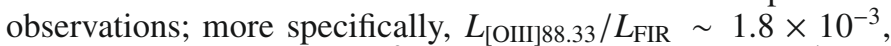

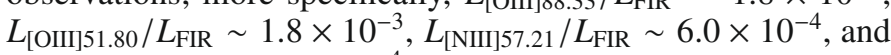
$L_{[\mathrm{NII}] 121.7} / L_{\mathrm{FIR}} \sim 2.7 \times 10^{-4}$ (Fischer et al. 1996; Colbert et al. 1999). The scale on the right hand side gives the minimum SFR corresponding the minimum $L_{\mathrm{FIR}}$ by using the SFR- $L_{\mathrm{FIR}}$ relationship given in Kennicutt (1999). Figure 9 suggests that we can detect all the fine-structure emission lines needed to infer the metallicity in luminous infrared galaxies $\left(L_{\mathrm{FIR}} \sim 10^{11}-10^{12} L_{\odot}\right)$ at redshifts up to $z \sim 0.4$. The detection to somewhat higher 


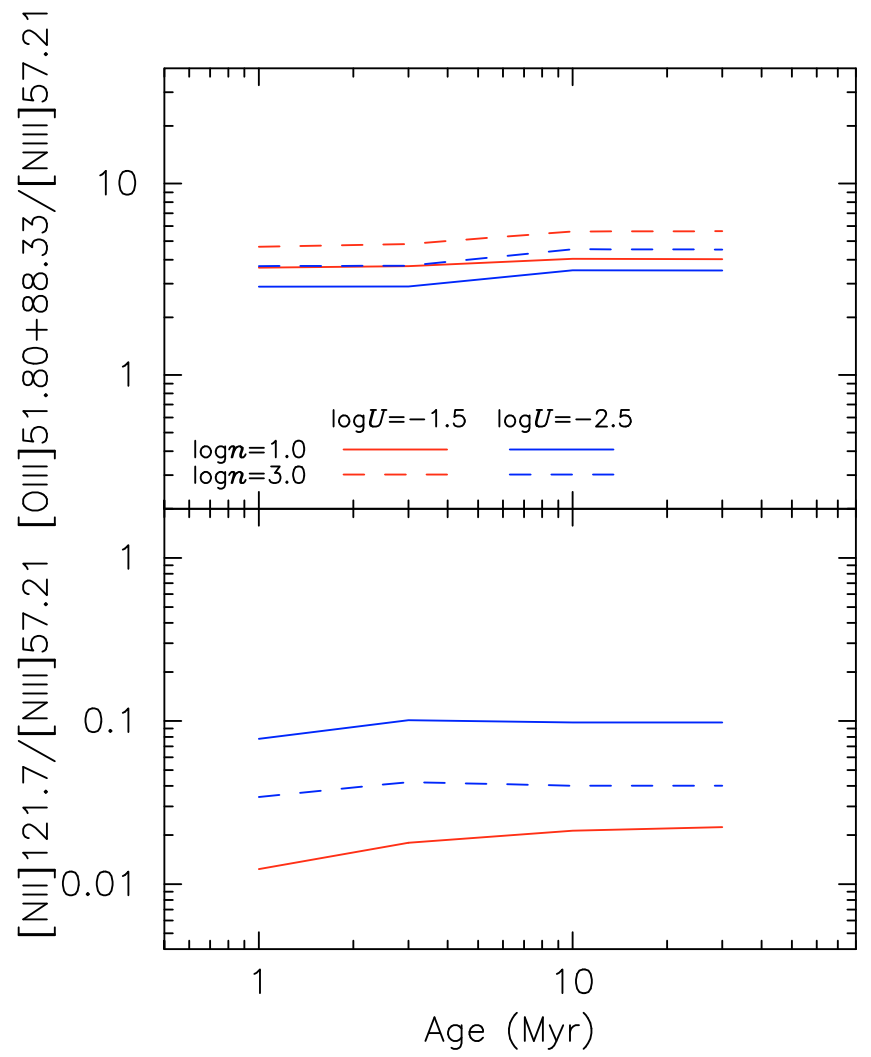

Fig. 8. Dependence of the predicted emission-line flux ratios ([OIII]51.80+[OIII]88.33)/[NIII]57.21 (upper panel) and [NII]121.7/ [NIII]57.21 (lower panel) on the age of the stellar population used in the photoionization model calculations. Models with constant starformation history and with solar metallicity are displayed. The meanings of lines are the same as in Fig. 3. In the lower panel, models with $n_{\mathrm{H}}=10^{3} \mathrm{~cm}^{-3}$ and $U=10^{-1.5}$ are not plotted, because the flux of the [NII]121.7 is too faint for those parameters. The displayed ranges of emission-line flux ratios in the upper and lower panels are the same as those in Fig. 5 and the lower panel of Fig. 6, respectively.

redshift is restricted to ULIRGs $\left(L_{\mathrm{FIR}}>10^{12} L_{\odot}\right)$. On the other hand, SAFARI boarded on SPICA will drastically expand the detectability, as shown in Fig. 10. By using SAFARI, the diagnostic fine-structure lines will be detectable for star-forming galaxies at $z \sim 1$ or at even higher redshift. In Fig. 10 we adopt the SAFARI sensitivity given by Swinyard (2008) with a scaling of sensitivity for a conservative $3.0 \mathrm{~m}$ aperture. Actually the redshift limit may be limited not by the instrument sensitivity, but by the wavelength coverage, although the instrument design of SAFARI is not yet completely fixed.

\section{Summary}

We investigated gas metallicity diagnostics with infrared finestructure emission lines, based on detailed photoionization model calculations. The main results are:

- Some fine-structure lines arise both in HII regions and PDRs and thus only emission lines arising within HII regions (not in PDRs) should be used as good metallicity diagnostics.

- Emission-line flux ratios that consist of [OIII]51.80, [NIII]57.21, and [OIII]88.33 provide good metallicity diagnostics; especially the flux ratio of ([OIII]51.80+ $[\mathrm{OIII}] 88.33) /[\mathrm{NIII}] 57.21$ is sensitive to the gas metallicity with a small dependence on the gas density.

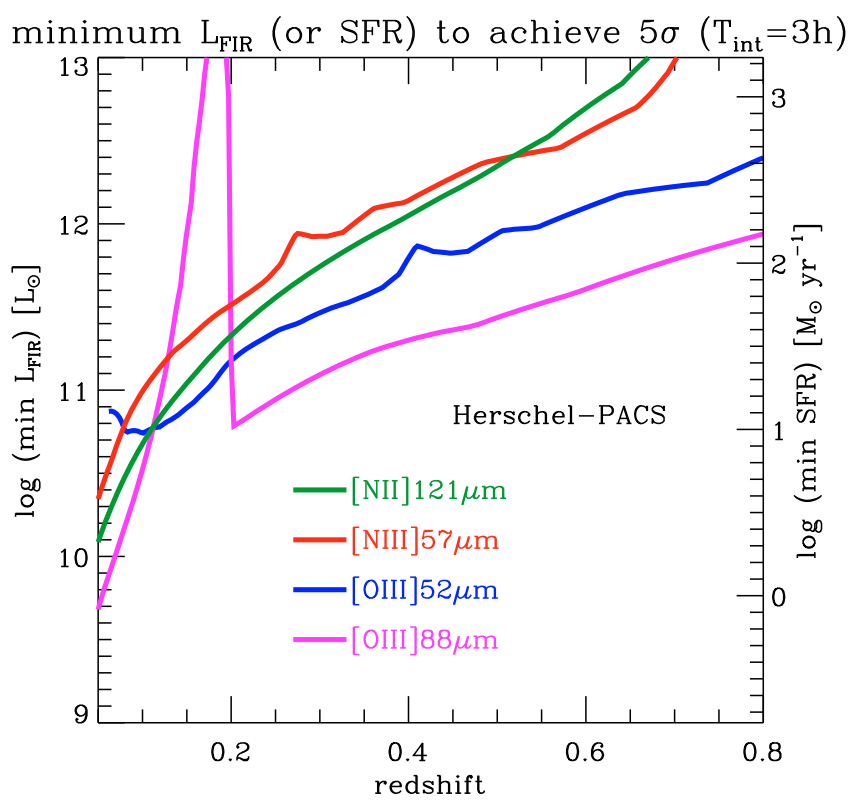

Fig. 9. Detectability with PACS on Herschel of some diagnostic far-IR fine-structure lines discussed in this paper, which can be used to measure the gas metallicity, ionization parameter, and density in galaxies, as a function of redshift. The left hand scale gives minimum $L_{\mathrm{FIR}}$ to achieve a $5 \sigma$ detection of each line with an exposure time of 3 hours. The right hand axis shows the minimum star-formation rate for the line detectability corresponding to the minimum $L_{\mathrm{FIR}}$ based on the relation given in Kennicutt (1999). Green, red, blue, and magenta lines denote [NII]121.7, [NIII] 57.21, [OIII]51.80, and [OIII] 88.33 , respectively.

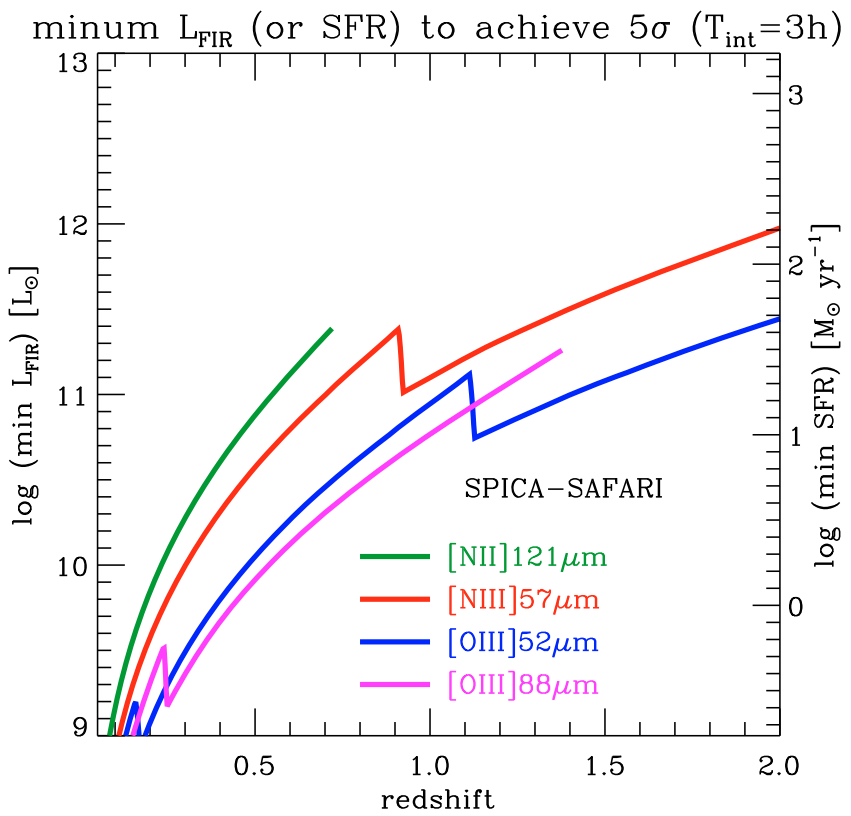

Fig. 10. Same as Fig. 9 but for the detectability with SAFARI boarded on SPICA.

- To correct for the effects of the ionization parameter on the infrared metallicity diagnostics, flux ratios of [NeII]12.81/ [NeIII]15.55 and [NII]121.7/[NIII]57.21 are useful.

- The detection of those infrared fine-structure lines is feasible, up to $z \sim 0.4$ with using Herschel/PACS and even up to $z \sim 1$ or more with using SPICA/SAFARI. 
Because the infrared fine-structure emission lines are not affected by the dust extinction, the gas-metallicity diagnostics presented in this paper are useful to assess the chemical properties in dust-obscured populations such as infrared galaxies, which are very important in terms of the galaxy evolution. Some of the infrared fine-structure emission lines from high- $z$ galaxies will be apparently exciting targets also for ALMA.

Acknowledgements. We thank Malcolm Walmsley, Luigi Spinoglio, Yasuhiro Shioya, and the anonymous referee for useful comments and suggestions. T.N. acknowledges financial supports through the Research Promotion Award of Ehime University and the Kurata Grant from the Kurata Memorial Hitachi Science and Technology Foundation. A.M. and R.M. acknowledge support from the Italian National Institute for Astrophysics through a PRIN/INAF 2008 grant.

\section{References}

Aannestad, P. A., \& Emery, R. J. 2003, A\&A, 406, 155

Abel, N. P. 2006, MNRAS, 368, 1949

Abel, N. P., Ferland, G. J., Shaw, G., \& van Hoof, P. A. M. 2005, ApJS, 161, 65 Baldwin, J. A., Ferland, G. J., Martin, P. G., et al. 1991, ApJ, 374, 580 Bastian, N., Emsellem, E., Kissler-Patig, M., \& Maraston, C. 2006, A\&A, 445, 471

Bastian, N., Trancho, G., Konstantoloulos, I. S., \& Miller, B. W. 2009, ApJ, 701, 607

Brooks, A. M., Governato, F., Booth, C. M., et al. 2007, ApJ, 655, L17

Caputi, K. I., Lilly, S. J., Aussel, H., et al. 2008, ApJ, 680, 939

Cardelli, J. A., Savage, B. D., Bruhweiler, F. C., et al. 1991, ApJ, 377, L57

Chiappini, C., Romano, D., \& Matteucci, F. 2003, MNRAS, 339, 63

Cid Fernandes, R., Leão, J. R. S., \& Rodrigues-Lacerda, R. 2003, MNRAS, 340, 29

Colbert, J. W., Malkan, M. A., Clegg, P. E., et al. 1999, ApJ, 511, 721

Cowie, L. L., \& Songaila, A. 1986, ARA\&A, 24, 499

Crawford, M. K., Genzel, R., Townes, C. H., et al. 1985, ApJ, 291, 755

Dale, D. A., Smith, J. D. T., Armus, L., et al. 2006, ApJ, 646, 161

de Rossi, M. E., Tissera, P. B., \& Scannapieco, C. 2007, MNRAS, 374, 323

Erb, D. K., Shapley, A. E., Pettini, M., et al. 2006, ApJ, 644, 813

Ferland, G. J. 2008, Hazy: A Brief Introduction to Cloudy version 08 (Lexington:

Univ. Kentucky Dept. Phys. Astron.)

Ferland, G. J., \& Mushotzky, R. F. 1984, ApJ, 286, 42

Ferland, G. J., Korista, K. T., Verner, D. A., et al. 1998, PASP, 110, 761

Finlator, K., \& Davé, R. 2008, MNRAS, 385, 2181

Fischer, J., Shier, L. M., Luhman, M. L., et al. 1996, A\&A, 315, L97

Garnett, D. R., Edmunds, M. G., Henry, R. B. C., et al. 2004, AJ, 128, 2772

Gorjian, V., Turner, J. L., \& Beck, S. C. 2001, ApJ, 554, L29
Groves, B. A., Dopita, M. A., \& Sutherland, R. S. 2004, ApJS, 153, 75 Hailey-Dunsheath, S., Nikola, T., Stacey, G. J., et al. 2010, ApJ, 714, L162 Hamann, F., \& Ferland, G. J. 1993, ApJ, 418, 11

Heiles, C. 1994, ApJ, 436, 720

Iono, D., Yun, M. S., Elvis, M., et al. 2006, ApJ, 645, L97

Jiang, L., Fan, X., \& Vestergaard, M. 2008, ApJ, 679, 962

Kewley, L. J., \& Dopita, M. A. 2002, ApJS, 142, 35

Kobayashi, C., Springel, V., \& White, S. D. M. 2007, MNRAS, 376, 1465

Lee, H., Skillman, E. D., Cannon, J. M., et al. 2006, ApJ, 647, 970

Le Floc'h, E., Papovich, C., Dole, H., et al. 2005, ApJ, 632, 169

Leitherer, C., Schaerer, D., Goldader, J. D., et al. 1999, ApJS, 123, 3

Liu, X. -W., Barlow, M. J., Cohen, M., et al. 2001, MNRAS, 323, 343

Maier, C., Lilly, S. J., Carollo, C. M., et al. 2006, ApJ, 639, 858

Maiolino, R., Cox, P., Caselli, P., et al. 2005, A\&A, 440, L5

Maiolino, R., Nagao, T., Grazian, A., et al. 2008, A\&A, 488, 463

Maiolino, R., Caselli, P., Nagao, T., et al. 2009, A\&A, 500, L1

Mannucci, F., Cresci, G., Maiolino, R., et al. 2009, MNRAS, 398, 1915

Mannucci, F., Cresci, G., Maiolino, R., et al. 2010, MNRAS, 408, 2115

McCall, M. L., Rybski, P. M., \& Shields, G. A. 1985, ApJS, 57, 1

Nagao, T., Maiolino, R., \& Marconi, A. 2006a, A\&A, 459, 85

Nagao, T., Marconi, A. \& Maiolino, R. 2006b, A\&A, 447, 157

Nakagawa, T., \& the SPICA team 2009, in SPICA Joint European/Japanese

Workshop, ed. A. M. Heras, B. M. Swinyard, K. G. Isaak, \& J. R. Goicoechea (EDP Sciences), 1001

Origlia, L., Ranalli, P., Comastri, A., \& Maiolino, R. 2004, ApJ, 606, 862

Panuzzo, P., Bressan, A., Granato, G. L., Silva, L., \& Danese, L. 2003, A\&A, 409, 99

Pettini, M., Shapley, A. E., Steidel, C. C., et al. 2001, ApJ, 554, 981

Petuchowski, S. J., \& Bennett, C. L. 1993, ApJ, 405, 591

Pilyugin, L. S., Thuan, T. X., \& Vílchez, J. M. 2003, A\&A, 397, 487

Poglitsch, A., Waelkens, C., Geis, N., et al. 2010, A\&A, 518, L2

Rodríguez Zaurín, J., Tadhunter, C. N., \& González Delgado, R. M. 2010, MNRAS, 403, 1317

Rupke, D. S. N., Veilleux, S., \& Baker, A. J. 2008, ApJ, 674, 172

Santini, P., Maiolino, R., Magnelli, B., et al. 2010, A\&A, 518, L154

Savaglio, S., Glazeblook, K., Le Borgue, D., et al. 2005, ApJ, 635, 260

Soifer, B. T., Helou, G., \& Werner, M. 2008, ARA\&A, 46, 201

Stacey, G. J., Geis, N., Genzel, R., et al. 1991, ApJ, 373, 423

Swinyard, B. 2008, The 37th COSPAR Scientific Assembly, 37, 3104

Swinyard, B., Nakagawa, T., Merken, P., et al. 2009, Experimental Astronomy, 23,193

Tremonti, C. A., Heckman, T. M., Kauffmann, G., et al. 2004, ApJ, 613, 898

Vacca, W. D., Johnson, K. E., \& Conti, P. S. 2002, AJ, 123, 772

van Zee, L., Salzer, J. J., \& Haynes, M. P. 1998, ApJ, 497, L1

Verma, A., Lutz, D., Sturm, E., et al. 2003, A\&A, 403, 829

Wu, Y., Bernard-Salas, J., Charmandaris, V., et al. 2008, ApJ, 673, 193

York, D. G., Adelman, J., Anderson, J. E., Jr., et al. 2000, AJ, 120, 1579

Zaritsky, D., Kennicutt, R. C., Jr., \& Huchra, J. P. 1994, ApJ, 420, 87 